FACTA UNIVERSITATIS (NIŠ)

Ser. Math. Inform. Vol. 36, No 2 (2021), 275-291

https://doi.org/10.22190/FUMI200604021A

Original Scientific Paper

\title{
HOMOTHETIC MOTIONS VIA GENERALIZED BICOMPLEX NUMBERS
}

\author{
Ferdağ Kahraman Aksoyak ${ }^{1}$, Sıddıka Özkaldı Karakuş ${ }^{2}$ \\ 1 Ahi Evran University, Faculty of Education, \\ Division of Elementary Mathematics Education, 40100 Kırşehir, Turkey \\ 2 Bilecik Şeyh Edebali University, Faculty of Arts and Science, \\ Department of Mathematics, 11000 Bilecik, Turkey
}

\begin{abstract}
In this paper, by using the matrix representation of generalized bicomplex numbers, we have defined the homothetic motions on some hypersurfaces in four dimensional generalized linear space $\mathbb{R}_{\alpha \beta}^{4}$. Also, for some special cases we have given some examples of homothetic motions in $\mathbb{R}^{4}$ and $\mathbb{R}_{2}^{4}$ and obtained some rotational matrices, too. Therefore, we have investigated some applications about kinematics of generalized bicomplex numbers.
\end{abstract}

Key words: Bicomplex number, Generalized Bicomplex numbers, Homothetic motion.

\section{Introduction}

In the middle of the 1800s, several mathematicians discussed the problem of whether a number system extended the field of complex numbers. In 1843, Sir William Rowan Hamilton defined a number system which is called quaternions in four dimensional space. Although quaternions and complex numbers have a lot of similar properties, quaternions are not commutative with respect to multiplication. So, in 1892, a new number system called bicomplex numbers was discovered by Corrado Segre [13]. Unlike quaternions, bicomplex numbers are commutative four dimensional real algebra.

Received June 4, 2020, accepted February 26, 2021

Communicated by Mića Stanković

Corresponding Author: F. Kahraman Aksoyak, Ahi Evran University, Faculty of Education, Division of Elementary Mathematics Education, 40100 Kirşehir, Turkey | E-mail: ferdag.aksoyak@ahievran.edu.tr

2010 Mathematics Subject Classification. Primary 30G35; Secondary 53A17, 53B50

(C) 2021 by University of Niš, Serbia | Creative Commons License: CC BY-NC-ND 
The set of bicomplex numbers denoted by $\mathbb{C}_{2}$ is defined as:

$\mathbb{C}_{2}=\left\{x=x_{1} 1+x_{2} i+x_{3} j+x_{4} i j: i^{2}=-1, j^{2}=-1, i j=j i, x_{k} \in \mathbb{R}, 1 \leq k \leq 4\right\}$.

Any $x$ bicomplex number can be rewritten as $x=z_{1}+j z_{2}$, where $z_{1}=x_{1}+$ $i x_{2}$ and $z_{2}=x_{3}+i x_{4}$ are complex numbers and $j$ is a different imaginer unit from the imaginer unit $i$ satisfied $j^{2}=-1$ and $i j=j i$. Hence, we can perceive a bicomplex number as a complex number whose components are complex numbers. There are some applications of bicomplex numbers on the algebra, geometry and analysis. A first theory of differentiability in $\mathbb{C}_{2}$ was developed by Price in [12]. Özkaldı Karakuş and Kahraman Aksoyak defined generalized bicomplex numbers and gave some algebraic properties. Also, they showed that some hypersurfaces in four dimensional generalized linear space are Lie groups by using generalized bicomplex number product and obtained Lie algebras of these Lie groups [10].

Kabadayı and Yaylı defined the homothetic motions with the help of bicomplex numbers in $\mathbb{R}^{4}[5]$. They showed that this homothetic motion under some conditions holds all of the properties in [14], [15]. Alkaya studied the homothetic motion with bicomplex numbers in $\mathbb{R}^{4}$ and $\mathbb{R}_{2}^{4}[1]$.

In this paper, by using the matrix representation of generalized bicomplex numbers, we shall define the homothetic motions on some hypersurfaces in four dimensional generalized linear space $\mathbb{R}_{\alpha \beta}^{4}$. Also, for some special cases we shall give some examples of homothetic motions in $\mathbb{R}^{4}$ and $\mathbb{R}_{2}^{4}$ and obtain some rotational matrices, too. Therefore, we shall investigate some applications about kinematics of generalized bicomplex numbers.

\section{Preliminaries}

In this section we give some basic concepts about generalized bicomplex numbers defined by Özkaldı Karakuş and Kahraman Aksoyak [10].

A generalized bicomplex number $x$ is defined as follows:

$$
x=x_{1} 1+x_{2} i+x_{3} j+x_{4} i j,
$$

where $x_{k}$ for $1 \leq k \leq 4$ are real numbers and the basis $\{1, i, j, i j\}$ holds $i^{2}=-\alpha$, $j^{2}=-\beta,(i j)^{2}=\alpha \beta, i j=j i, \alpha, \beta \in \mathbb{R}$. The set of generalized bicomplex numbers is denoted by $\mathbb{C}_{\alpha \beta}$. For any two generalized bicomplex numbers $x=x_{1}+x_{2} i+x_{3} j+x_{4} i j$ and $y=y_{1}+y_{2} i+y_{3} j+y_{4} i j$, addition and multiplication are as follows:

$$
x+y=\left(x_{1}+y_{1}\right)+\left(x_{2}+y_{2}\right) i+\left(x_{3}+y_{3}\right) j+\left(x_{4}+y_{4}\right) i j,
$$

$x \cdot y=\left(x_{1} y_{1}-\alpha x_{2} y_{2}-\beta x_{3} y_{3}+\alpha \beta x_{4} y_{4}\right)+\left(x_{1} y_{2}+x_{2} y_{1}-\beta x_{3} y_{4}-\beta x_{4} y_{3}\right) i$

(2.1) $\quad+\left(x_{1} y_{3}+x_{3} y_{1}-\alpha x_{2} y_{4}-\alpha x_{4} y_{2}\right) j+\left(x_{1} y_{4}+x_{4} y_{1}+x_{2} y_{3}+x_{3} y_{2}\right) i j$

and the scalar multiplication of an element in $\mathbb{C}_{\alpha \beta}$ by a real number $c$ is as:

$$
c x=c x_{1} 1+c x_{2} i+c x_{3} j+c x_{4} i j .
$$


Hence, by means of these elementary arithmetic operations on $\mathbb{C}_{\alpha \beta}$, we have two important results. $\mathbb{C}_{\alpha \beta}$ is a four dimensional real vector space with respect to addition and scalar multiplication and it is a commutative real algebra according to generalized bicomplex number product.

Let us consider the following set of matrices

$$
Q_{\alpha \beta}=\left\{M_{x}=\left(\begin{array}{cccc}
x_{1} & -\alpha x_{2} & -\beta x_{3} & \alpha \beta x_{4} \\
x_{2} & x_{1} & -\beta x_{4} & -\beta x_{3} \\
x_{3} & -\alpha x_{4} & x_{1} & -\alpha x_{2} \\
x_{4} & x_{3} & x_{2} & x_{1}
\end{array}\right): x_{i} \in \mathbb{R}, 1 \leq i \leq 4\right\},
$$

where the set $Q_{\alpha \beta}$ is a vector space with matrix addition and scalar matrix product and it is an algebra together with matrix product. The algebras $\mathbb{C}_{\alpha \beta}$ and $Q_{\alpha \beta}$ are isomorphic. The isomorphism between two algebras is defined as:

$$
\begin{gathered}
h: \mathbb{C}_{\alpha \beta} \rightarrow Q_{\alpha \beta}, \\
h\left(x_{1} 1+x_{2} i+x_{3} j+x_{4} i j\right)=\left(\begin{array}{cccc}
x_{1} & -\alpha x_{2} & -\beta x_{3} & \alpha \beta x_{4} \\
x_{2} & x_{1} & -\beta x_{4} & -\beta x_{3} \\
x_{3} & -\alpha x_{4} & x_{1} & -\alpha x_{2} \\
x_{4} & x_{3} & x_{2} & x_{1}
\end{array}\right) .
\end{gathered}
$$

With the help of this isomorphism, any generalized bicomplex number in $\mathbb{C}_{\alpha \beta}$ can be represent by a matrix in $Q_{\alpha \beta}$. Moreover, it is possible to express the generalized bicomplex number product which has been given by (2.1) by matrix product, that is,

$$
x \cdot y=\left(\begin{array}{cccc}
x_{1} & -\alpha x_{2} & -\beta x_{3} & \alpha \beta x_{4} \\
x_{2} & x_{1} & -\beta x_{4} & -\beta x_{3} \\
x_{3} & -\alpha x_{4} & x_{1} & -\alpha x_{2} \\
x_{4} & x_{3} & x_{2} & x_{1}
\end{array}\right)\left(\begin{array}{l}
y_{1} \\
y_{2} \\
y_{3} \\
y_{4}
\end{array}\right) .
$$

A generalized bicomplex number can be rewritten as $x=\left(x_{1}+x_{2} i\right)+\left(x_{3}+x_{4} i\right) j$. There are three kinds of conjugations for generalized bicomplex numbers. They are given as follows:

$$
\begin{aligned}
& x^{t_{1}}=\left[\left(x_{1}+x_{2} i\right)+\left(x_{3}+x_{4} i\right) j\right]^{t_{1}}=\left(x_{1}-x_{2} i\right)+\left(x_{3}-x_{4} i\right) j, \\
& x^{t_{2}}=\left[\left(x_{1}+x_{2} i\right)+\left(x_{3}+x_{4} i\right) j\right]^{t_{2}}=\left(x_{1}+x_{2} i\right)-\left(x_{3}+x_{4} i\right) j, \\
& x^{t_{3}}=\left[\left(x_{1}+x_{2} i\right)+\left(x_{3}+x_{4} i\right) j\right]^{t_{3}}=\left(x_{1}-x_{2} i\right)-\left(x_{3}-x_{4} i\right) j,
\end{aligned}
$$

where $x^{t_{1}}, x^{t_{2}}$ and $x^{t_{3}}$ denote the conjugations of $x$ with respect to $i, j$ and both $i$ and $j$, respectively. Also we can compute

$$
\begin{aligned}
& x \cdot x^{t_{1}}=\left(x_{1}^{2}+\alpha x_{2}^{2}-\beta x_{3}^{2}-\alpha \beta x_{4}^{2}\right)+2\left(x_{1} x_{3}+\alpha x_{2} x_{4}\right) j, \\
& x \cdot x^{t_{2}}=\left(x_{1}^{2}-\alpha x_{2}^{2}+\beta x_{3}^{2}-\alpha \beta x_{4}^{2}\right)+2\left(x_{1} x_{2}+\beta x_{3} x_{4}\right) i, \\
& x \cdot x^{t_{3}}=\left(x_{1}^{2}+\alpha x_{2}^{2}+\beta x_{3}^{2}+\alpha \beta x_{4}^{2}\right)+2\left(x_{1} x_{4}-x_{2} x_{3}\right) i j .
\end{aligned}
$$




\section{One Parameter Homothetic Motion}

Let the fixed space and the moving space be $R_{0}$ and $R$., respectively. The oneparameter homothetic motion of $R_{0}$ with respect to $R$ is denoted by $R_{0} / R$. This motion is obtained by the following transformation

$$
\left[\begin{array}{c}
X \\
1
\end{array}\right]=\left[\begin{array}{cc}
h A & C \\
0 & 1
\end{array}\right]\left[\begin{array}{c}
X_{0} \\
1
\end{array}\right]
$$

or it can be expressed as

$$
X=B X_{0}+C
$$

in which $X_{0}$ and $X$ are the position vectors of the same point in $R_{0}$ and $R$, respectively and $B=h A$. Also, $h, A$ and $C$ are continuously differentiable functions depend on the real parameter $t$, where $h: I \subset \mathbb{R} \rightarrow \mathbb{R}, t \rightarrow h(t)$ is called homothetic scale of the motion, $A$ is a real quasi-orthogonal matrix that holds $A^{T} \varepsilon A=\varepsilon(\varepsilon$ is a signature matrix according to metric), $C$ is the translation matrix. To avoid the case of affine transformation we suppose that $h$ is not constant and to avoid the cases of pure translation and pure rotation we also assume that $\frac{d(h A)}{d t} \neq 0$ and $\frac{d C}{d t} \neq 0[2]$.

\section{Pole Points and Pole Curves of the Homothetic Motion}

If we take the derivative of (3.1) with respect to $t$, we obtain the following equality

$$
\dot{X}=\dot{B} X_{0}+\dot{C}+B \dot{X}_{0},
$$

where $\dot{X}$ is the absolute velocity, $\dot{B} X_{0}+\dot{C}$ is the sliding velocity and $B \dot{X}_{0}$ is the relative velocity of the point $X_{0}$. The points at which the sliding velocity of the motion vanishes at all time $t$ are called pole points of the motion in $R_{0}$. In that case, to determine the pole points of the motion, we solve the following equality

$$
\dot{B} X_{0}+\dot{C}=0 \text {. }
$$

For more details see[2].

\section{Homothetic Motions on Some Hypersurfaces via Generalized Bicomplex Numbers}

In this section we have defined the homothetic motions on some hypersurfaces at $\mathbb{R}_{\alpha \beta}^{4}$ with the help of generalized bicomplex numbers and given some examples about the homothetic motions. 


\subsection{Homothetic Motion on Hypersurface $M_{1}$}

Let us consider the hypersurface $M_{1}$ as follows:

$$
M_{1}=\left\{x=\left(x_{1}, x_{2}, x_{3}, x_{4}\right) \in \mathbb{R}_{\alpha \beta}^{4}: x_{1} x_{3}+\alpha x_{2} x_{4}=0, x \neq 0\right\} .
$$

By using generalized bicomplex numbers, $M_{1}$ can be rewritten as:

$$
M_{1}=\left\{x=x_{1} 1+x_{2} i+x_{3} j+x_{4} i j \in \mathbb{R}_{\alpha \beta}^{4}: x_{1} x_{3}+\alpha x_{2} x_{4}=0, x \neq 0\right\},
$$

or the hypersurface $M_{1}$ can be expressed by using the matrix representiation of generalized bicomplex numbers

$$
\tilde{M}_{1}=\left\{M_{x}=\left(\begin{array}{cccc}
x_{1} & -\alpha x_{2} & -\beta x_{3} & \alpha \beta x_{4} \\
x_{2} & x_{1} & -\beta x_{4} & -\beta x_{3} \\
x_{3} & -\alpha x_{4} & x_{1} & -\alpha x_{2} \\
x_{4} & x_{3} & x_{2} & x_{1}
\end{array}\right): x_{1} x_{3}+\alpha x_{2} x_{4}=0, x \neq 0\right\},
$$

where $M_{x}$ is the matrix representiation of generalized bicomplex number $x$. The metric on hypersurface $M_{1}$ is defined by $g_{1}(x, x)=x \cdot x^{t_{1}}=x_{1}^{2}+\alpha x_{2}^{2}-\beta x_{3}^{2}-\alpha \beta x_{4}^{2}$ and the norm of any element $x$ on $M_{1}$ is defined by $\|x\|=\sqrt{\left|g_{1}(x, x)\right|}=\sqrt{\mid x \cdot x^{t_{1} \mid}}$. This metric is Riemannian or pseudo-Riemannian metric on four dimensional generalized linear space $\mathbb{R}_{\alpha \beta}^{4}$ and for some special cases, it coincides with four dimensional Euclidean space $\mathbb{R}^{4}$ or four dimensional pseudo-Euclidean space $\mathbb{R}_{2}^{4}$.

Proposition 5.1. There are following properties about the norm on the hypersurface $M_{1}$.

i) For $x, y \in M_{1},\|x \cdot y\|=\|x\|\|y\|$,

ii) $\|x\|^{4}=\operatorname{det}\left(M_{x}\right)$.

Proof. These properties can be easily seen with direct calculations.

Corollary 5.1. A unit generalized bicomplex number on the hypersurface $M_{1}$ determines a rotation motion.

Proof. It is obvious from Proposition 5.1.

Theorem 5.1. $M_{1}$ is a commutative Lie group.

Proof. The proof can be found in [10].

Let us denote the set of unit generalized bicomplex numbers on $M_{1}$ by $M_{1}^{*} . M_{1}^{*}$ is as:

$$
\begin{aligned}
M_{1}^{*} & =\left\{x \in M_{1}: g_{1}(x, x)=1\right\} \\
& =\left\{x \in M_{1}: x_{1}^{2}+\alpha x_{2}^{2}-\beta x_{3}^{2}-\alpha \beta x_{4}^{2}=1\right\} .
\end{aligned}
$$


Theorem 5.2. $M_{1}^{*}$ is Lie subgroup of $M_{1}$.

Proof. The proof can be found in [10].

Let $\gamma$ be a curve on $M_{1}$. In that case, it can be expressed as

$$
\begin{array}{lll}
\gamma & : \quad I \subset \mathbb{R} \rightarrow M_{1} \\
t & \rightarrow \quad \gamma(t)=\gamma_{1}(t)+\gamma_{2}(t) i+\gamma_{3}(t) j+\gamma_{4}(t) i j, \gamma_{1}(t) \gamma_{3}(t)+\alpha \gamma_{2}(t) \gamma_{4}(t)=0
\end{array}
$$

Then the matrix $B$ corresponding to the curve $\gamma$ is obtained as follows:

$$
B=M_{\gamma(t)}=\left[\begin{array}{cccc}
\gamma_{1}(t) & -\alpha \gamma_{2}(t) & -\beta \gamma_{3}(t) & \alpha \beta \gamma_{4}(t) \\
\gamma_{2}(t) & \gamma_{1}(t) & -\beta \gamma_{4}(t) & -\beta \gamma_{3}(t) \\
\gamma_{3}(t) & -\alpha \gamma_{4}(t) & \gamma_{1}(t) & -\alpha \gamma_{2}(t) \\
\gamma_{4}(t) & \gamma_{3}(t) & \gamma_{2}(t) & \gamma_{1}(t)
\end{array}\right]
$$

Now by using this matrix $B$, we can define the one parameter motion on $M_{1}$ at $\mathbb{R}_{\alpha \beta}^{4}$.

Definition 5.1. Let $R_{0}$ and $R$ be the fixed space and the motional space at $\mathbb{R}_{\alpha \beta}^{4}$. In that case, the one-parameter motion of $R_{0}$ with respect to $R$ is denoted by $R_{0} / R$. Then the one-parameter motion on $M_{1}$ is defined by

$$
\left[\begin{array}{c}
X \\
1
\end{array}\right]=\left[\begin{array}{cc}
B & C \\
0 & 1
\end{array}\right]\left[\begin{array}{c}
X_{0} \\
1
\end{array}\right]
$$

or it can be expressed as

$$
X=B X_{0}+C
$$

where $B$ is the matrix associated with the curve $\gamma(t)$ on the hypersurface $M_{1}, C$ is the $4 \times 1$ real matrix depends on a real parameter $t, X$ and $X_{0}$ are the position vectors of any point at $\mathbb{R}_{\alpha \beta}^{4}$ respectively in $R$ and $R_{0}$.

Theorem 5.3. The equation given by (5.2) determines a homothetic motion on $M_{1}$.

Proof. Since the curve $\gamma$ lies on $M_{1}$, it does not pass through the origin. So, the matrix given by (5.1) can be expressed as:

$$
B=M_{\gamma(t)}=h\left[\begin{array}{cccc}
\frac{\gamma_{1}(t)}{h} & \frac{-\alpha \gamma_{2}(t)}{h} & \frac{-\beta \gamma_{3}(t)}{h} & \frac{\alpha \beta \gamma_{4}(t)}{h} \\
\frac{\gamma_{2}(t)}{h} & \frac{\gamma_{1}(t)}{h} & \frac{-\beta \gamma_{4}(t)}{h} & \frac{-\beta \gamma_{3}(t)}{h} \\
\frac{\gamma_{3}(t)}{h} & \frac{-\alpha \gamma_{4}(t)}{h} & \frac{\gamma_{1}(t)}{h} & \frac{-\alpha \gamma_{2}(t)}{h} \\
\frac{\gamma_{4}(t)}{h} & \frac{\gamma_{3}(t)}{h} & \frac{\gamma_{2}(t)}{h} & \frac{\gamma_{1}(t)}{h}
\end{array}\right]=h A,
$$

where $h: I \subset \mathbb{R} \rightarrow \mathbb{R}, t \rightarrow h(t)=\|\gamma(t)\|=\sqrt{\gamma_{1}^{2}+\alpha \gamma_{2}^{2}-\beta \gamma_{3}^{2}-\alpha \beta \gamma_{4}^{2}}$. Because of $\gamma(t) \in M_{1}, \gamma_{1}(t) \gamma_{3}(t)+\alpha \gamma_{2}(t) \gamma_{4}(t)=0$. By using this equality, we obtain that 
the matrix $A$ in (5.3) is a real quasi-orthogonal matrix. In that case it satisfies $A^{T} \varepsilon A=\varepsilon$ and $\operatorname{det} A=1$, where $\varepsilon$ is the signature matrix corresponding to metric $g_{1}$ is as:

$$
\varepsilon=\left[\begin{array}{cccc}
1 & 0 & 0 & 0 \\
0 & \alpha & 0 & 0 \\
0 & 0 & -\beta & 0 \\
0 & 0 & 0 & -\alpha \beta
\end{array}\right]
$$

Hence $A, h$ and $C$ are a real quasi-orthogonal matrix, the homothetic scale of the motion and the translation vector, respectively. So the equation (5.2) is a homothetic motion.

Remark 5.1. The norm of $\gamma \in \mathbb{R}_{\alpha \beta}^{4}$ is found as $\|\gamma(t)\|=\sqrt{\left|\gamma_{1}^{2}+\alpha \gamma_{2}^{2}-\beta \gamma_{3}^{2}-\alpha \beta \gamma_{4}^{2}\right|}$. We assume that $\gamma_{1}^{2}+\alpha \gamma_{2}^{2}-\beta \gamma_{3}^{2}-\alpha \beta \gamma_{4}^{2}>0$ in this paper.

Corollary 5.2. Let $\gamma(t)$ be a curve on $M_{1}^{*}$. Then one-parameter motion on $M_{1}$ given by (5.2) is a general motion consists of a rotation and a translation.

Proof. We assume that $\gamma(t)$ is a curve on $M_{1}^{*}$. Then $\gamma_{1}^{2}+\alpha \gamma_{2}^{2}-\beta \gamma_{3}^{2}-\alpha \beta \gamma_{4}^{2}=1$. In that case the matrix $B$ given by (5.1) becomes a real-quasi orthogonal matrix, that is, it satisfies $B^{T} \varepsilon B=\varepsilon$ and $\operatorname{det} B=1$. This completes the proof.

Theorem 5.4. Let $\gamma(t)$ be a unit velocity curve and its tangent vector $\dot{\gamma}(t)$ be on $M_{1}$. Then the derivative of the matrix $B$ is a real quasi-orthogonal matrix.

Proof. We suppose that $\gamma(t)$ be a unit velocity curve. Then $\dot{\gamma}_{1}^{2}+\alpha \dot{\gamma}_{2}^{2}-\beta \dot{\gamma}_{3}^{2}-$ $\alpha \beta \dot{\gamma}_{4}^{2}=1$. Also, since the tangent vector of $\gamma$ is on $M_{1}$, it implies that $\dot{\gamma}_{1}(t) \dot{\gamma}_{3}(t)+$ $\alpha \dot{\gamma}_{2}(t) \dot{\gamma}_{4}(t)=0$. Thus $\dot{B}^{T} \varepsilon \dot{B}=\varepsilon$ and $\operatorname{det} \dot{B}=1$.

Theorem 5.5. Let $\gamma(t)$ be a unit velocity curve and its tangent vector $\dot{\gamma}(t)$ be on $M_{1}$. Then the motion is a regular motion and it is independent of $h$.

Proof. From Theorem 5.4, $\operatorname{det} \dot{B}=1$ and thus the value of $\operatorname{det} \dot{B}$ is independent of $h$.

Theorem 5.6. Let $\gamma(t)$ be a unit velocity curve whose the position vector and tangent vector are on $M_{1}$. Then the pole points of the motion given by (5.2) are $X_{0}=-\dot{B}^{-1} \dot{C}$.

Proof. Since the position vector of the curve $\gamma$ is on $M_{1}$, from Theorem 5.3, the equation (5.2) becomes a homothetic motion. Also, because of $\gamma(t)$ is a unit velocity curve and $\dot{\gamma}(t) \in M_{1}$, from Theorem $5.4 \operatorname{det} \dot{B}=1$ and it implies that there is only one solution of the equation (4.1). Then the pole points of the motion given by (5.2) are obtained as $X_{0}=-\dot{B}^{-1} \dot{C}$. 
Corollary 5.3. Let $\gamma(t)$ be a unit velocity curve whose the position vector and tangent vector are on $M_{1}$. The pole point associated with each $t$ - instant in $R_{0}$ is the rotation by the matrix $\dot{B}^{-1}$ of the speed vector of translation vector at the opposite direction $(-\dot{C})$.

Proof. From Theorem 5.4, the matrix $\dot{B}$ is a real quasi-orthogonal matrix. Then the matrix $\dot{B}^{-1}$ is quasi-orthogonal matrix, too. This completes the proof.

Now we will give various examples of the homothetic motions on $M_{1}$ according to the situations of real numbers $\alpha$ and $\beta$.

Example 5.1. For $\alpha=\beta=1, M_{1}$ becomes a hypersurface in $\mathbb{R}_{2}^{4}$. Let $\gamma: I \subset \mathbb{R} \rightarrow M_{1} \subset$ $\mathbb{R}_{2}^{4}$ be a curve given by

$$
\gamma(t)=h(t)\left(\begin{array}{c}
\cosh (a t) \cos (b t)+\cosh (a t) \sin (b t) i \\
-\sinh (a t) \sin (b t) j+\sinh (a t) \cos (b t) i j
\end{array}\right),
$$

where $a$ and $b$ are real numbers. By using (5.1) and (5.4), the matrix $B$ associated with the curve $\gamma$ becomes a homothetic matrix, where $h: I \subset \mathbb{R} \rightarrow \mathbb{R}$ is a homothetic scale. Also, if we take as $h(t)=1$ in (5.4), then $\gamma$ is a curve on $M_{1}^{*}$ and the matrix $B$ determines a rotation matrix in $\mathbb{R}_{2}^{4}$. In (5.4), if we choose as $h(t)=1, a=0$ and $b=1$, then we get

$$
\gamma(t)=\cos t+i \sin t .
$$

By using (5.1) and (5.5), we get the matrix $B$ as follows:

$$
B=\left(\begin{array}{cccc}
\cos t & -\sin t & 0 & 0 \\
\sin t & \cos t & 0 & 0 \\
0 & 0 & \cos t & -\sin t \\
0 & 0 & \sin t & \cos t
\end{array}\right)
$$

where $B$ is a rotational matrix in $\mathbb{R}_{2}^{4}$. Since this curve given by (5.5) is unit speed curve and its tangent vector belongs to $M_{1}$, the derivation of the above matrix $\dot{B}$ is a real quasiorthogonal matrix, too. Then it is a rotational matrix in $\mathbb{R}_{2}^{4}$. Similarly, in (5.4) if we take as $h(t)=1, a=1$ and $b=0$, then we get

$$
\gamma(t)=\cosh t+i j \sinh t .
$$

By using (5.1) and (5.6), we have the matrix $B$ as follows:

$$
B=\left(\begin{array}{cccc}
\cosh t & 0 & 0 & \sinh t \\
0 & \cosh t & -\sinh t & 0 \\
0 & -\sinh t & \cosh t & 0 \\
\sinh t & 0 & 0 & \cosh t
\end{array}\right)
$$

where $B$ is a rotational matrix in $\mathbb{R}_{2}^{4}$.

Example 5.2. For $\alpha=1, \beta=-1, M_{1}$ is a hypersurface in $\mathbb{R}^{4}$. Let $\gamma: I \subset \mathbb{R} \rightarrow M_{1} \subset \mathbb{R}^{4}$ be a curve given by

$$
\gamma(t)=h(t)\left(\begin{array}{c}
\cos (a t) \cos (b t)+\cos (a t) \sin (b t) i \\
-\sin (a t) \sin (b t) j+\sin (a t) \cos (b t) i j
\end{array}\right)
$$


where $a$ and $b$ are real numbers. By using (5.1) and (5.7), the matrix representation of the curve $\gamma$ is a homothetic matrix, in here $h: I \subset \mathbb{R} \rightarrow \mathbb{R}$ is a homothetic scale. Also, for $h(t)=1, \gamma$ becomes a spherical curve on $M_{1}$, that is, $\gamma(t) \in M_{1}^{*}=M_{1} \cap S^{3}$. The matrix representation of it is a rotation matrix in $\mathbb{R}^{4}$. Even, if we take as $h(t)=1, a=0, b=1$, then

$$
\gamma(t)=\cos t+i \sin t
$$

From (5.1) and (5.8), by determining the matrix representation of the above curve, we obtain

$$
B=\left(\begin{array}{cccc}
\cos t & -\sin t & 0 & 0 \\
\sin t & \cos t & 0 & 0 \\
0 & 0 & \cos t & -\sin t \\
0 & 0 & \sin t & \cos t
\end{array}\right)
$$

This matrix is a general rotational matrix in $\mathbb{R}^{4}$ which is defined by Moore [8]. Also, from Theorem 5.4, $\dot{B}$ is a real orthogonal matrix, too.

Example 5.3. For $\alpha=\beta=-1$, the hypersurface $M_{1}$ lies in $\mathbb{R}_{2}^{4}$ and the following curve lies on $M_{1}$

$$
\gamma(t)=h(t)\left(\begin{array}{c}
\cosh (a t) \cosh (b t)+\cosh (a t) \sinh (b t) i \\
+\sinh (a t) \sinh (b t) j+\cosh (a t) \sinh (b t) i j
\end{array}\right),
$$

in which $a$ and $b$ are real numbers. From (5.1) and (5.9), the matrix $B$ according to the curve $\gamma$ is a homothetic matrix, where $h: I \subset \mathbb{R} \rightarrow \mathbb{R}$ is a homothetic scale. Also, if we take as $h(t)=1$, then $\gamma$ lies on $M_{1}^{*}$ and the matrix $B$ gives a rotation matrix in $\mathbb{R}_{2}^{4}$.

\subsection{Homothetic Motion on Hypersurface $M_{2}$}

Let us consider the hypersurface $M_{2}$ as follows:

$$
M_{2}=\left\{x=\left(x_{1}, x_{2}, x_{3}, x_{4}\right) \in \mathbb{R}_{\alpha \beta}^{4}: x_{1} x_{2}+\beta x_{3} x_{4}=0, x \neq 0\right\} .
$$

By using the generalized bicomplex numbers, $M_{2}$ can be rewritten as:

$$
M_{2}=\left\{x=x_{1} 1+x_{2} i+x_{3} j+x_{4} i j \in \mathbb{R}_{\alpha \beta}^{4}: x_{1} x_{2}+\beta x_{3} x_{4}=0, x \neq 0\right\},
$$

or the hypersurface $M_{2}$ can be expressed by using the matrix representiation of generalized bicomplex numbers

$$
\tilde{M}_{2}=\left\{M_{x}=\left(\begin{array}{cccc}
x_{1} & -\alpha x_{2} & -\beta x_{3} & \alpha \beta x_{4} \\
x_{2} & x_{1} & -\beta x_{4} & -\beta x_{3} \\
x_{3} & -\alpha x_{4} & x_{1} & -\alpha x_{2} \\
x_{4} & x_{3} & x_{2} & x_{1}
\end{array}\right): x_{1} x_{2}+\beta x_{3} x_{4}=0, x \neq 0\right\},
$$

where $M_{x}$ is the matrix representiation of the generalized bicomplex number $x$ on $M_{2}$. The metric on hypersurface $M_{2}$ is defined by $g_{2}(x, x)=x \cdot x^{t_{2}}=x_{1}^{2}-\alpha x_{2}^{2}+$ $\beta x_{3}^{2}-\alpha \beta x_{4}^{2}$ and the norm of any element $x$ on $M_{2}$ is given by $\|x\|=\sqrt{\left|g_{2}(x, x)\right|}=$ $\sqrt{\mid x \cdot x^{t_{2} \mid}}$. This metric is Riemannian or pseudo-Riemannian metric on four dimensional generalized linear space $\mathbb{R}_{\alpha \beta}^{4}$ and for some special cases, it coincides with four dimensional Euclidean space $\mathbb{R}^{4}$ or four dimensional pseudo-Euclidean space $\mathbb{R}_{2}^{4}$. 
Proposition 5.2. There are following properties about the norm on the hypersurface $M_{2}$.

i) For $x, y \in M_{2},\|x \cdot y\|=\|x\|\|y\|$,

ii) $\|x\|^{4}=\operatorname{det}\left(M_{x}\right)$.

Proof. These properties can be easily seen with directly calculations.

Corollary 5.4. A unit generalized bicomplex number on the hypersurface $M_{2}$ determines a rotation motion.

Proof. It is obvious from Proposition 5.2.

Theorem 5.7. $M_{2}$ is a commutative Lie group.

Proof. The proof can be found in [10].

Let us denote the set of unit generalized bicomplex number on $M_{2}$ by $M_{2}^{*} . M_{2}^{*}$ is given as:

$$
\begin{aligned}
M_{2}^{*} & =\left\{x \in M_{2}: g_{2}(x, x)=1\right\} \\
& =\left\{x \in M_{2}: x_{1}^{2}-\alpha x_{2}^{2}+\beta x_{3}^{2}-\alpha \beta x_{4}^{2}=1\right\} .
\end{aligned}
$$

Theorem 5.8. $M_{2}^{*}$ is Lie subgroup of $M_{2}$.

Proof. The proof can be found in [10].

Let $\gamma$ be a curve on $M_{2}$. In that case, it can be expressed as:

$$
\begin{array}{lll}
\gamma & : \quad I \subset \mathbb{R} \rightarrow M_{2} \\
t & \rightarrow \quad \gamma(t)=\gamma_{1}(t)+\gamma_{2}(t) i+\gamma_{3}(t) j+\gamma_{4}(t) i j, \gamma_{1}(t) \gamma_{2}(t)+\beta \gamma_{3}(t) \gamma_{4}(t)=0 .
\end{array}
$$

Then the matrix $B$ corresponding to the curve $\gamma$ is given as follows:

$$
B=M_{\gamma(t)}=\left[\begin{array}{cccc}
\gamma_{1}(t) & -\alpha \gamma_{2}(t) & -\beta \gamma_{3}(t) & \alpha \beta \gamma_{4}(t) \\
\gamma_{2}(t) & \gamma_{1}(t) & -\beta \gamma_{4}(t) & -\beta \gamma_{3}(t) \\
\gamma_{3}(t) & -\alpha \gamma_{4}(t) & \gamma_{1}(t) & -\alpha \gamma_{2}(t) \\
\gamma_{4}(t) & \gamma_{3}(t) & \gamma_{2}(t) & \gamma_{1}(t)
\end{array}\right]
$$

Now by using this matrix $B$, we can define the one parameter motion on $M_{2}$ at $\mathbb{R}_{\alpha \beta}^{4}$.

Definition 5.2. Let $R_{0}$ and $R$ be the fixed space and the motional space at $\mathbb{R}_{\alpha \beta}^{4}$. In that case, the one-parameter motion of $R_{0}$ with respect to $R$ is denoted by $R_{0} / R$. Then the one-parameter motion on $M_{2}$ is given by

$$
\left[\begin{array}{c}
X \\
1
\end{array}\right]=\left[\begin{array}{cc}
B & C \\
0 & 1
\end{array}\right]\left[\begin{array}{c}
X_{0} \\
1
\end{array}\right],
$$


or it can be expressed as

$$
X=B X_{0}+C
$$

where $B$ is the matrix associated with the curve $\gamma(t)$ on the hypersurface $M_{2}, C$ is the $4 \times 1$ real matrix depends on a real parameter $t, X$ and $X_{0}$ are the position vectors of any point at $\mathbb{R}_{\alpha \beta}^{4}$ respectively in $R$ and $R_{0}$.

Theorem 5.9. The equation given by (5.11) determines a homothetic motion on $M_{2}$.

Proof. Since the curve $\gamma$ is on $M_{2}$, it does not pass through the origin. So, the matrix given by (5.10) can be expressed as:

$$
B=M_{\gamma(t)}=h\left[\begin{array}{cccc}
\frac{\gamma_{1}(t)}{h} & \frac{-\alpha \gamma_{2}(t)}{h} & \frac{-\beta \gamma_{3}(t)}{h} & \frac{\alpha \beta \gamma_{4}(t)}{h} \\
\frac{\gamma_{2}(t)}{h} & \frac{\gamma_{1}(t)}{h} & \frac{-\beta \gamma_{4}(t)}{h} & \frac{-\beta \gamma_{3}(t)}{h} \\
\frac{\gamma_{3}(t)}{h} & \frac{-\alpha \gamma_{4}(t)}{h} & \frac{\gamma_{1}(t)}{h} & \frac{-\alpha \gamma_{2}(t)}{h} \\
\frac{\gamma_{4}(t)}{h} & \frac{\gamma_{3}(t)}{h} & \frac{\gamma_{2}(t)}{h} & \frac{\gamma_{1}(t)}{h}
\end{array}\right]=h A,
$$

where $h: I \subset \mathbb{R} \rightarrow \mathbb{R}, t \rightarrow h(t)=\|\gamma(t)\|=\sqrt{\gamma_{1}^{2}-\alpha \gamma_{2}^{2}+\beta \gamma_{3}^{2}-\alpha \beta \gamma_{4}^{2}}$. Since $\gamma(t) \in M_{2}, \gamma_{1}(t) \gamma_{2}(t)+\beta \gamma_{3}(t) \gamma_{4}(t)=0$. By using this equality, we obtain that the matrix $A$ in (5.12) is a real quasi-orthogonal matrix. In that case it satisfies $A^{T} \varepsilon A=\varepsilon$ and $\operatorname{det} A=1$, where $\varepsilon$ is the signature matrix corresponding to metric $g_{2}$ given by

$$
\varepsilon=\left[\begin{array}{cccc}
1 & 0 & 0 & 0 \\
0 & -\alpha & 0 & 0 \\
0 & 0 & \beta & 0 \\
0 & 0 & 0 & -\alpha \beta
\end{array}\right]
$$

Hence $A, h$ and $C$ are a real quasi-orthogonal matrix, the homothetic scale of the motion and the translation vector, respectively. So the equation (5.11) determines a homothetic motion.

Remark 5.2. The norm of the curve $\gamma \in \mathbb{R}_{\alpha \beta}^{4}$ is found as

$\|\gamma(t)\|=\sqrt{\left|\gamma_{1}^{2}-\alpha \gamma_{2}^{2}+\beta \gamma_{3}^{2}-\alpha \beta \gamma_{4}^{2}\right|}$. We assume that $\gamma_{1}^{2}-\alpha \gamma_{2}^{2}+\beta \gamma_{3}^{2}-\alpha \beta \gamma_{4}^{2}>0$ in this paper.

Corollary 5.5. Let $\gamma(t)$ be a curve on $M_{2}^{*}$. Then one-parameter motion on $M_{2}$ given by (5.11) is a general motion consists of a rotation and a translation.

Proof. We assume that $\gamma(t)$ is a curve on $M_{2}^{*}$. Then $\gamma_{1}^{2}-\alpha \gamma_{2}^{2}+\beta \gamma_{3}^{2}-\alpha \beta \gamma_{4}^{2}=1$. In that case the matrix $B$ in (5.11) becomes a real-quasi orthogonal matrix, that is, it satisfies $B^{T} \varepsilon B=\varepsilon$ and $\operatorname{det} B=1$. This completes the proof.

Theorem 5.10. Let $\gamma(t)$ be a unit velocity curve and its tangent vector $\dot{\gamma}(t)$ be on $M_{2}$. Then the derivative of the matrix $B$ is a real quasi-orthogonal matrix. 
Proof. We suppose that $\gamma(t)$ be a unit velocity curve then $\dot{\gamma}_{1}^{2}-\alpha \dot{\gamma}_{2}^{2}+\beta \dot{\gamma}_{3}^{2}-\alpha \beta \dot{\gamma}_{4}^{2}=$ 1. Also since the tangent vector of the curve $\gamma$ is on $M_{2}$, we have $\dot{\gamma}_{1}(t) \dot{\gamma}_{2}(t)+$ $\beta \dot{\gamma}_{3}(t) \dot{\gamma}_{4}(t)=0$. Thus $\dot{B}^{T} \varepsilon \dot{B}=\varepsilon$ and $\operatorname{det} \dot{B}=1$.

Theorem 5.11. Let $\gamma(t)$ be a unit velocity curve and its tangent vector $\dot{\gamma}(t)$ be on $M_{2}$. Then the motion is a regular motion and it is independent of $h$.

Proof. From Theorem 5.10, $\operatorname{det} \dot{B}=1$ and thus the value of $\operatorname{det} \dot{B}$ is independent of $h$.

Theorem 5.12. Let $\gamma(t)$ be a unit velocity curve whose the position vector and tangent vector are on $M_{2}$. Then the pole points of the motion given by (5.11) are $X_{0}=-\dot{B}^{-1} \dot{C}$.

Proof. Since the position vector of the curve $\gamma$ is on $M_{2}$, from Theorem 5.9, the equation (5.11) becomes a homothetic motion. Also, because of $\gamma(t)$ is a unit velocity curve and $\dot{\gamma}(t) \in M_{2}$, from Theorem $5.10 \operatorname{det} \dot{B}=1$ and it implies that there is only one solution of the equation (4.1). Then the pole points of the motion given by (5.11) are found as $X_{0}=-\dot{B}^{-1} \dot{C}$.

Corollary 5.6. Let $\gamma(t)$ be a unit velocity curve whose the position vector and tangent vector are on $M_{2}$. The pole point associated with each $t$ - instant in $R_{0}$ is the rotation by the matrix $\dot{B}^{-1}$ of the speed vector of translation vector at the opposite direction $(-\dot{C})$.

Proof. From Theorem 5.10, the matrix $\dot{B}$ is a real quasi-orthogonal matrix. Then the matrix $\dot{B}^{-1}$ is quasi-orthogonal matrix, too. This completes the proof.

Now we will give various examples of the homothetic motions on $M_{2}$ according to the situations of real numbers $\alpha$ and $\beta$.

Example 5.4. For $\alpha=\beta=1, M_{2}$ becomes a hypersurface in $\mathbb{R}_{2}^{4}$. Let $\gamma: I \subset \mathbb{R} \rightarrow M_{2} \subset$ $\mathbb{R}_{2}^{4}$ be a curve as:

$$
\gamma(t)=h(t)\left(\begin{array}{c}
\cosh (a t) \cos (b t)-\sinh (a t) \sin (b t) i \\
+\cosh (a t) \sin (b t) j+\sinh (a t) \cos (b t) i j
\end{array}\right)
$$

where $a$ and $b$ are real numbers. By using (5.10) and (5.13), the matrix $B$ is a homothetic matrix and $h: I \subset \mathbb{R} \rightarrow \mathbb{R}$ is a homothetic scale. Also, for $h(t)=1$, the matrix $B$ becomes a rotation matrix in $\mathbb{R}_{2}^{4}$.

Example 5.5. For $\alpha=-1, \beta=1, M_{2}$ is a hypersurface in $\mathbb{R}^{4}$. Let us consider the curve $\gamma: I \subset \mathbb{R} \rightarrow M_{2} \subset \mathbb{R}^{4}$ as follows:

$$
\gamma(t)=h(t)\left(\begin{array}{c}
\cos (a t) \cos (b t)-\sin (a t) \sin (b t) i \\
+\cos (a t) \sin (b t) j+\sin (a t) \cos (b t) i j
\end{array}\right)
$$

where $a$ and $b$ are real numbers. From (5.10) and (5.14) we obtain the matrix representation of the curve $\gamma$ and it determines a homothetic matrix, in here $h: I \subset \mathbb{R} \rightarrow \mathbb{R}$ is a homothetic scale. Also, for $h(t)=1, \gamma$ becomes a spherical curve on $M_{2}$, that is, $\gamma(t) \in M_{2}^{*}=M_{2} \cap S^{3}$. From Corollary 5.5, the matrix representation of it is a rotation matrix in $\mathbb{R}^{4}$. 
Example 5.6. For $\alpha=\beta=-1$, the hypersurface $M_{2}$ becomes a subset of $\mathbb{R}_{2}^{4}$ and the following curve lies on $M_{2}$

$$
\gamma(t)=h(t)\left(\begin{array}{c}
\cosh (a t) \cosh (b t)+\sinh (a t) \sinh (b t) i \\
+\cosh (a t) \sinh (b t) j+\sinh (a t) \cosh (b t) i j
\end{array}\right),
$$

in which $a$ and $b$ are real numbers. From (5.10) and (5.15), the matrix $B$ according to the curve $\gamma$ is a homothetic matrix, where $h: I \subset \mathbb{R} \rightarrow \mathbb{R}$ is a homothetic scale. Also, if we take as $h(t)=1$, then $\gamma$ lies on $M_{2}^{*}$ and the matrix $B$ gives a rotation matrix in $\mathbb{R}_{2}^{4}$.

\subsection{Homothetic Motion on Hypersurface $M_{3}$}

Let us consider the hypersurface $M_{3}$ as follows:

$$
M_{3}=\left\{x=\left(x_{1}, x_{2}, x_{3}, x_{4}\right) \in \mathbb{R}_{\alpha \beta}^{4}: x_{1} x_{4}-x_{2} x_{3}=0, x \neq 0\right\} .
$$

By using generalized bicomplex numbers, $M_{3}$ can be rewritten as:

$$
M_{3}=\left\{x=x_{1} 1+x_{2} i+x_{3} j+x_{4} i j \in \mathbb{R}_{\alpha \beta}^{4}: x_{1} x_{4}-x_{2} x_{3}=0, x \neq 0\right\},
$$

or the hypersurface $M_{3}$ can be expressed by using the matrix representiation of generalized bicomplex numbers

$$
\tilde{M}_{3}=\left\{M_{x}=\left(\begin{array}{cccc}
x_{1} & -\alpha x_{2} & -\beta x_{3} & \alpha \beta x_{4} \\
x_{2} & x_{1} & -\beta x_{4} & -\beta x_{3} \\
x_{3} & -\alpha x_{4} & x_{1} & -\alpha x_{2} \\
x_{4} & x_{3} & x_{2} & x_{1}
\end{array}\right): x_{1} x_{4}-x_{2} x_{3}=0, x \neq 0\right\} .
$$

where $M_{x}$ is the matrix representiation of generalized bicomplex number $x$ on $M_{3}$. The metric on hypersurface $M_{3}$ is defined by $g_{3}(x, x)=x \cdot x^{t_{3}}=x_{1}^{2}+\alpha x_{2}^{2}+\beta x_{3}^{2}+$ $\alpha \beta x_{4}^{2}$ and the norm of any element $x$ on $M_{3}$ is given by $\|x\|=\sqrt{\left|g_{3}(x, x)\right|}=$ $\sqrt{\mid x \cdot x^{t_{3} \mid}}$. This metric is Riemannian or pseudo-Riemannian metric on four dimensional generalized linear space $\mathbb{R}_{\alpha \beta}^{4}$ and for some special cases, it coincides four dimensional Euclidean space $\mathbb{R}^{4}$ or four dimensional pseudo-Euclidean space $\mathbb{R}_{2}^{4}$.

Proposition 5.3. There are following properties about the norms on the hypersurface $M_{3}$.

i) For $x, y \in M_{3},\|x \cdot y\|=\|x\|\|y\|$

ii) $\|x\|^{4}=\operatorname{det}\left(M_{x}\right)$

Proof. These properties can be easily seen with direct calculations.

Corollary 5.7. A unit generalized bicomplex number on the hypersurface $M_{3}$ determines a rotation motion.

Proof. It is obvious from Proposition 5.3. 
Theorem 5.13. $M_{3}$ is a commutative Lie group.

Proof. The proof can be found in [10].

Let us denote the set of unit generalized bicomplex number on $M_{3}$ by $M_{3}^{*} . M_{3}^{*}$ is given as:

$$
\begin{aligned}
M_{3}^{*} & =\left\{x \in M_{3}: g_{3}(x, x)=1\right\} \\
& =\left\{x \in M_{3}: x_{1}^{2}+\alpha x_{2}^{2}+\beta x_{3}^{2}+\alpha \beta x_{4}^{2}=1\right\} .
\end{aligned}
$$

Theorem 5.14. $M_{3}^{*}$ is Lie subgroup of $M_{3}$.

Proof. The proof can be found in [10].

Let $\gamma$ be a curve on $M_{3}$. In that case, it can be expressed as

$$
\begin{array}{lll}
\gamma & : & I \subset \mathbb{R} \rightarrow M_{3} \\
t & \rightarrow & \gamma(t)=\gamma_{1}(t)+\gamma_{2}(t) i+\gamma_{3}(t) j+\gamma_{4}(t) i j, \gamma_{1}(t) \gamma_{4}(t)-\gamma_{2}(t) \gamma_{3}(t)=0 .
\end{array}
$$

Then the matrix $B$ corresponding to the curve $\gamma$ is given as follows:

$$
B=M_{\gamma(t)}=\left[\begin{array}{cccc}
\gamma_{1}(t) & -\alpha \gamma_{2}(t) & -\beta \gamma_{3}(t) & \alpha \beta \gamma_{4}(t) \\
\gamma_{2}(t) & \gamma_{1}(t) & -\beta \gamma_{4}(t) & -\beta \gamma_{3}(t) \\
\gamma_{3}(t) & -\alpha \gamma_{4}(t) & \gamma_{1}(t) & -\alpha \gamma_{2}(t) \\
\gamma_{4}(t) & \gamma_{3}(t) & \gamma_{2}(t) & \gamma_{1}(t)
\end{array}\right]
$$

Now by using this matrix $B$, we can define the one parameter motion on $M_{3}$ at $\mathbb{R}_{\alpha \beta}^{4}$.

Definition 5.3. Let $R_{0}$ and $R$ be the fixed space and the motional space at $\mathbb{R}_{\alpha \beta}^{4}$. In that case, the one-parameter motion of $R_{0}$ with respect to $R$ is denoted by $R_{0} / R$. Then the one-parameter motion on $M_{3}$ is given by

$$
\left[\begin{array}{c}
X \\
1
\end{array}\right]=\left[\begin{array}{cc}
B & C \\
0 & 1
\end{array}\right]\left[\begin{array}{c}
X_{0} \\
1
\end{array}\right],
$$

or it can be expressed as

$$
X=B X_{0}+C
$$

where $B$ is the matrix associated with the curve $\gamma(t)$ on the hypersurface $M_{3}, C$ is the $4 \times 1$ real matrix depends on a real parameter $t, X$ and $X_{0}$ are the position vectors of any point at $\mathbb{R}_{\alpha \beta}^{4}$ respectively in $R$ and $R_{0}$, respectively.

Theorem 5.15. The equation given by (5.17) is a homothetic motion on $M_{3}$. 
Proof. Since the curve $\gamma$ is on $M_{3}$, it does not pass through the origin. So, the matrix given by (5.16) can be expressed as:

$$
B=M_{\gamma(t)}=h\left[\begin{array}{cccc}
\frac{\gamma_{1}(t)}{h} & \frac{-\alpha \gamma_{2}(t)}{h} & \frac{-\beta \gamma_{3}(t)}{h} & \frac{\alpha \beta \gamma_{4}(t)}{h} \\
\frac{\gamma_{2}(t)}{h} & \frac{\gamma_{1}(t)}{h} & \frac{-\beta \gamma_{4}(t)}{h} & \frac{-\beta \gamma_{3}(t)}{h} \\
\frac{\gamma_{3}(t)}{h} & \frac{-\alpha \gamma_{4}(t)}{h} & \frac{\gamma_{1}(t)}{h} & \frac{-\alpha \gamma_{2}(t)}{h} \\
\frac{\gamma_{4}(t)}{h} & \frac{\gamma_{3}(t)}{h} & \frac{\gamma_{2}(t)}{h} & \frac{\gamma_{1}(t)}{h}
\end{array}\right]=h A,
$$

where $h: I \subset \mathbb{R} \rightarrow \mathbb{R}, t \rightarrow h(t)=\|\gamma(t)\|=\sqrt{\gamma_{1}^{2}+\alpha \gamma_{2}^{2}+\beta \gamma_{3}^{2}+\alpha \beta \gamma_{4}^{2}}$. Because of $\gamma(t) \in M_{3}$, we have $\gamma_{1}(t) \gamma_{4}(t)-\gamma_{2}(t) \gamma_{3}(t)=0$. By using this equality, we obtain that the matrix $A$ in (5.18) is a real quasi-orthogonal matrix. In that case it satisfies $A^{T} \varepsilon A=\varepsilon$ and $\operatorname{det} A=1$, where $\varepsilon$ is the signature matrix corresponding to metric $g_{3}$ given by

$$
\varepsilon=\left[\begin{array}{cccc}
1 & 0 & 0 & 0 \\
0 & \alpha & 0 & 0 \\
0 & 0 & \beta & 0 \\
0 & 0 & 0 & \alpha \beta
\end{array}\right]
$$

Hence $A, h$ and $C$ are a real quasi-orthogonal matrix, the homothetic scale of the motion and the translation vector, respectively. So the equation (5.17) determines a homothetic motion.

Remark 5.3. The norm of the curve $\gamma \in \mathbb{R}_{\alpha \beta}^{4}$ is found as $\|\gamma(t)\|=\sqrt{\left|\gamma_{1}^{2}+\alpha \gamma_{2}^{2}+\beta \gamma_{3}^{2}+\alpha \beta \gamma_{4}^{2}\right|}$. We assume that $\gamma_{1}^{2}+\alpha \gamma_{2}^{2}+\beta \gamma_{3}^{2}+\alpha \beta \gamma_{4}^{2}>0$ in this paper.

Corollary 5.8. Let $\gamma(t)$ be a curve on $M_{3}^{*}$. Then one-parameter motion on $M_{3}$ given by (5.17) is a general motion consists of a rotation and a translation.

Proof. We assume that $\gamma(t)$ is a curve on $M_{3}^{*}$. Then $\gamma_{1}^{2}+\alpha \gamma_{2}^{2}+\beta \gamma_{3}^{2}+\alpha \beta \gamma_{4}^{2}=1$. In that case the matrix $B$ given by (5.16) becomes a real-quasi orthogonal matrix, that is, it satisfies $B^{T} \varepsilon B=\varepsilon$ and $\operatorname{det} B=1$. This completes the proof.

Theorem 5.16. Let $\gamma(t)$ be a unit velocity curve and its tangent vector $\dot{\gamma}(t)$ be on $M_{3}$. Then the derivative of the matrix $B$ is a real quasi-orthogonal matrix.

Proof. We suppose that $\gamma(t)$ be a unit velocity curve. Then $\dot{\gamma}_{1}^{2}+\alpha \dot{\gamma}_{2}^{2}+\beta \dot{\gamma}_{3}^{2}+\alpha \beta \dot{\gamma}_{4}^{2}=$ 1. Also since the tangent vector of the curve $\gamma$ is on $M_{3}$, we have $\dot{\gamma}_{1}(t) \dot{\gamma}_{4}(t)-$ $\dot{\gamma}_{2}(t) \dot{\gamma}_{3}(t)=0$. Thus $\dot{B}^{T} \varepsilon \dot{B}=\varepsilon$ and $\operatorname{det} \dot{B}=1$.

Theorem 5.17. Let $\gamma(t)$ be a unit velocity curve and its tangent vector $\dot{\gamma}(t)$ be on $M_{3}$. Then the motion is a regular motion and it is independent of $h$.

Proof. From Theorem 5.16, $\operatorname{det} \dot{B}=1$ and thus the value of $\operatorname{det} \dot{B}$ is independent of $h$. 
Theorem 5.18. Let $\gamma(t)$ be a unit velocity curve whose the position vector and tangent vector are on $M_{3}$. Then the pole points of the motion given by (5.17) are $X_{0}=-\dot{B}^{-1} \dot{C}$.

Proof. Since the position vector of the curve $\gamma$ is on $M_{3}$, from Theorem 5.15, the equation (5.17) is a homothetic motion. Also, because of $\gamma(t)$ is a unit velocity curve and $\dot{\gamma}(t) \in M_{3}$, from Theorem $5.16 \operatorname{det} \dot{B}=1$. Thus the equation (4.1) has only one solution. In that case the pole points of the motion are obtained as $X_{0}=-\dot{B}^{-1} \dot{C}$.

Corollary 5.9. Let $\gamma(t)$ be a unit velocity curve whose the position vector and tangent vector are on $M_{3}$. The pole point associated with each $t$ - instant in $R_{0}$ is the rotation by the matrix $\dot{B}^{-1}$ of the speed vector of translation vector at the opposite direction $(-\dot{C})$.

Proof. From Theorem 5.16, the matrix $\dot{B}$ is a real quasi-orthogonal matrix. Then the matrix $\dot{B}^{-1}$ is quasi-orthogonal matrix, too. This completes the proof.

Now we will give various examples of the homothetic motions on $M_{3}$ according to the situations of real numbers $\alpha$ and $\beta$.

Example 5.7. For $\alpha=\beta=1, M_{3}$ becomes a hypersurface in four dimensional Euclidean space $\mathbb{R}^{4}$. Let $\gamma: I \subset \mathbb{R} \rightarrow M_{3} \subset \mathbb{R}^{4}$ be a curve as:

$$
\gamma(t)=h(t)\left(\begin{array}{c}
\cos (a t) \cos (b t)+\cos (a t) \sin (b t) i \\
+\sin (a t) \cos (b t) j+\sin (a t) \sin (b t) i j
\end{array}\right)
$$

where $a$ and $b$ are real numbers. By using (5.16) and (5.19), the matrix $B$ associated with the curve $\gamma$ is a homothetic matrix, where $h: I \subset \mathbb{R} \rightarrow \mathbb{R}$ is a homothetic scale. Also, if we take as $h(t)=1$, then $\gamma$ is a curve on $M_{3}^{*}$. In that case it becomes a spherical curve lies on $M_{3}$ and the matrix $B$ becomes a rotation matrix in $\mathbb{R}^{4}$.

Example 5.8. For $\alpha=1, \beta=-1, M_{3}$ is a hypersurface in four dimensional Euclidean space $\mathbb{R}_{2}^{4}$. Let $\gamma: I \subset \mathbb{R} \rightarrow M_{3} \subset \mathbb{R}_{2}^{4}$ be a curve given by

$$
\gamma(t)=h(t)\left(\begin{array}{c}
\cosh (a t) \cos (b t)+\cosh (a t) \sin (b t) i \\
+\sinh (a t) \cos (b t) j+\sinh (a t) \sin (b t) i j
\end{array}\right),
$$

where $a$ and $b$ are real numbers. By using (5.16) and (5.20), the matrix representation of the curve $\gamma$ is a homothetic matrix, in here $h: I \subset \mathbb{R} \rightarrow \mathbb{R}$ is a homothetic scale. Also, for $h(t)=1, \gamma$ becomes a spherical curve on $M_{3}$, that is, $\gamma(t) \in M_{3}^{*}$. The matrix representation of it is a rotation matrix in $\mathbb{R}_{2}^{4}$.

Example 5.9. For $\alpha=\beta=-1$, the hypersurface $M_{3}$ is in four dimensional pseudoEuclidean space $\mathbb{R}_{2}^{4}$ and the following curve lies on $M_{3}$

$$
\gamma(t)=h(t)\left(\begin{array}{c}
\cosh (a t) \cosh (b t)+\cosh (a t) \sinh (b t) i \\
+\sinh (a t) \cosh (b t) j+\sinh (a t) \sinh (b t) i j
\end{array}\right),
$$

in which $a$ and $b$ are real numbers. From (5.16) and (5.21), the matrix $B$ according to the curve $\gamma$ is a homothetic matrix, where $h: I \subset \mathbb{R} \rightarrow \mathbb{R}$ is a homothetic scale. Also, if we take as $h(t)=1$, then $\gamma$ lies on $M_{3}^{*}$ and the matrix $B$ gives a rotation matrix in $\mathbb{R}_{2}^{4}$. 


\section{REF E R E N C E S}

1. D. Alkaya: Homothetic motions with bicomplex numbers. Master's Thesis, Dumlupinar University, Institute of Science, Kütahya, 2013.

2. H. H. HacisalihoĞLU: On the rolling of one curve or surface upon another. Proc. R. Irish Acad. 71 (1971), 13-17.

3. M. JAFARI and Y. YAYLI : Rotation in four dimensions via generalized Hamilton operators. Kuwait J. Sci. 40 (2013), 67-79.

4. M. JAFARI and Y. YAYLI : Generalized quaternions and rotation in 3-space $\mathbb{E}_{\alpha \beta}^{3}$. TWMS J. Pure Appl. Math. 6 (2015), 224-232.

5. H. KABADAYI and Y. YAYLI : Homothetic motion at $E^{4}$ with bicomplex numbers. Adv. Appl. Clifford Algebr., 21 (2011), 541-546.

6. F. Kahraman Aksoyak and Y. YAyli: Homothetic motions and surfaces in $\mathbb{E}^{4}$. Bull. Malays. Math. Sci. Soc. 38 (2015), 259-269.

7. F. Kahraman Aksoyak and Y. YAYLI: Homothetic motions and Lie groups in $\mathbb{E}_{2}^{4}$. J. Dyn. Syst. Geom. Theo. 11 (2013), 23-38.

8. C. L. E. Moore : Surfaces of rotation in a space of four dimensions. Ann. of Math. 21 (1919), 81-93.

9. S. ÖZKALDI KARAKUş and Y. YAYLI : Bicomplex number and tensor product surfaces in $\mathbb{R}_{2}^{4}$. Ukrainian Mathematical Journal, 64 (2012), 344-355.

10. S. Özkaldi karakuş and F. Kahraman Aksoyak: Generalized bicomplex numbers and Lie groups. Adv. Appl. Clifford Algebr., 25 (2015), 943-963.

11. S. ÖZKaldi Karakuş and Y. YAYLI : Tensor product surfaces in $\mathbb{R}^{4}$ and Lie groups. Bull. Malays. Math. Sci.Soc. (2) 33 (2010), 69-77.

12. G. B. PRICE : An Introduction to Multicomplex Spaces and Functions, Marcel Dekker, Inc., New York, 1990.

13. C. SEGRE: Le rappresentazioni reali delle forme complesse e gli enti iperalgebrici. Math. Ann. 40 (1892), 1-25.

14. Y. YAYLI : Homothetic motions at $E^{4}$. Mech. Mach. Theory, 27 (1992), 303-305.

15. Y. YAYLI and B. BüKCÜ : Homothetic motions at $E^{8}$ with cayley numbers. Mech. Mach. Theory, 30 (1995), 417-420. 